\title{
A Proposal for Scalable 5G New Radio Frames with Enhanced Throughput
}

\author{
Hedaia Ghannam and Izzat Darwazeh \\ Department of Electrical and Electronics Engineering \\ University College London \\ London, United Kingdom \\ E-Mail: hedaia.ghannam.15@ucl.ac.uk,i.darwazeh@ucl.ac.uk
}

\begin{abstract}
This paper investigates, for the first time, a new scheme for increasing the data rates of $5 G$ new radio (NR), through replacing the orthogonal frequency division multiplexing (OFDM) signal format with a multi-carrier signal with more than 20\% improved spectral efficiency. Such signal format is known as spectrally efficient frequency division multiplexing (SEFDM), where the subcarriers' orthogonality is intentionally violated to increase spectral efficiency compared to OFDM. A new method to generate continuous cyclic prefix (CP) for SEFDM signals is reported. Furthermore, the work details a newly proposed system structure capable of generating both continuous CP and special pilot signals as part of the SEFDM frame. The behaviour of such system is investigated by comparing the error rates and throughput to 5G OFDM-based systems. Results show that the use of SEFDM signals can increase the spectral efficiency by at least $22.8 \%$ relative to OFDM, at the expense of a power penalty below $1 \mathrm{~dB}$.
\end{abstract}

\section{INTRODUCTION}

A key feature of the $5 \mathrm{G}$ new radio (NR) frame structure is its scalability, where the orthogonal frequency division multiplexing (OFDM) subcarriers spacing and subframe duration can change to support diverse use cases (i.e. higher data rates and reduced latency) [1] [2]. Boosting up the data rates is achieved by using a higher modulation order and/or transmit over higher bandwidth. Yet, another degree of freedom can be achieved in optimizing the $5 \mathrm{G}$ frame structure by using spectrally efficient frequency division multiplexing (SEFDM), where subcarrier spacing can be further reduced beyond orthogonality [3]. The advantage of 5G NR SEFDM-based frame is interpereted into an increase of the system throughput without increasing the modulation order, where more subcarriers will occupy the same bandwidth of a radio frame when compared to OFDM [4].

The intentional violation of orthogonality in SEFDM results in inter-carrier interference (ICI), which is dependent on the compression factor $\alpha \leq 1$ [5]. Notwithstanding, the use of SEFDM signals yields better peak to average power ratio (PAPR) characteristics [6] [7] and enhanced error performance, when compared to OFDM of the same throughput [8] [9]. Furthermore, the efficacy of SEFDM was proven experimentally for wireless [9], optical/mm-wave [10], VLC [11], very high speed optical systems [12] [13] and in internet of things (IoT) scenarios [14].
Conventionally, the ICI-impaired SEFDM signals have been studied in the frequency domain, as the main idea behind these signals is to reduce subcarrier's frequency spacing. However, the structure of SEFDM signals in the time domain has not been investigated well, until the recent work of [15] and what will be presented in this paper. In [15], SEFDM time domain characteristics inspired the development of a new pilot format, where the pilot signals are sent on advance of SEFDM symbols over orthogonally spaced subcarriers as an OFDM symbol. The pilot symbol uses the same number of subcarriers and the same frequency spacing of the SEFDM signals used to transmit data. As a result, a simple and accurate channel frequency response (CFR) estimation is guaranteed at the expense of slightly increased pilot duration overhead.

In this work, and for the first time, a new feature of SEFDM is realised in the time domain, which relates to the cyclic prefix (CP). What has essentially attracted the use of cyclic prefix (CP) instead of guard band (GB), in OFDM, is that CP maintains the signal continuity [16]. The continuity of CP transfers the circular convolution in time domain into multiplication in the frequency domain and this allows the employement of a one-tap equalizer in channel equalisation [16]. For SEFDM, the generation of continuous CP is found to be different than that of OFDM as will be shown later.

SEFDM signal generation by means of a single or multiple inverse fast Fourier transform (IFFT) has been already implemented in [17]. However, the capability of generating a 5G SEFDM-based frame via a single IFFT is more challenging for two reasons: i) the requirement of ICI-free pilot generation to estimate the CFR ; ii) the generation of continuous CP SEFDM signals. In this work, first, we will show the generation of a 5G SEFDM-based frame via a single reconfigurable IFFT. Thereafter, the efficiency of such a system is validated by comparing its error results and throughput to a 5G OFDMbased system.

\section{5G SEFDM-BASED FRAME}

SEFDM is a multi-carrier multiplexing technique, where symbols are generated similarly to OFDM. For an SEFDM symbol $x(t)$ with $N$ subcarriers, we take $\mathbf{z}=\left\{z_{0}, z_{1}, . ., z_{N-1}\right\}, \mathbf{z} \in \mathbb{C}^{N \times 1}$ to be the complex 
baseband symbols of symbol duration $T_{s}$, to be modulated by the SEFDM symbol's subcarriers, such as

$$
x(t)=\frac{1}{\sqrt{T}} \sum_{n=0}^{N-1} z_{n} \exp (j 2 \pi n \triangle f t), \quad 0 \leq t \leq T
$$

where $T$ is the SEFDM signal duration and is equal to $N \times T_{s}$, while $\triangle f$ is the frequency spacing between subcarriers. In contrast to OFDM, the SEFDM subcarriers are not orthogonal and $\triangle f=\alpha / T$, where $\alpha \leq 1$ is the compression factor.

The digital version of (1) is obtained by sampling the signal at least at the Nyquist rate, to allow reconstruction of the signal from its samples at the receiver. This implies that $Q \geq N$ samples are required [8]. The sampled version $x(q T / N)$ of (1), presented by the vector $\mathbf{x} \in \mathbb{C}^{Q \times 1}$ is

$$
\mathbf{x}=\frac{1}{\sqrt{Q}} \sum_{n=0}^{N-1} z_{n} \exp \left(j 2 \pi \alpha \frac{q n}{Q}\right), 0 \leq q \leq Q-1,
$$

where the factor $1 / \sqrt{Q}$ in (2) is employed for normalization purposes.

If the number of subcarriers for the case of OFDM $(\alpha=1)$ is given by $N_{o}$, then, due to spacing compression in SEFDM, more subcarriers will occupy the same bandwidth. The relation is given by $N=\left\lfloor N_{o} / \alpha\right\rfloor$, where $\lfloor$.$\rfloor denotes the floor$ operation. Resultantly, the data rate increases by a factor of $(1-\alpha) / \alpha \times 100 \%$.

In the following, the resource grid design is explained first, then the generation of SEFDM signals for NR-5G frames is implemented.

\section{A. Resource Grid Design}

In the recent 3 GPP standards (release $14 \& 15$ ) [1] [2], the standard numberology (subcarriers spacing and symbol length) of the $5 \mathrm{G} \mathrm{NR}$ is one radio frame of length $10 \mathrm{~ms}$ consisting of 10 subframes, each of length $1 \mathrm{~ms}$. Each subframe consists of an OFDM subcarrier spacing dependent number of slots, as it goes from one up to eight slots per subframe. Each slot consists of 14 OFDM symbols (12 OFDM symbols in case of extended $\mathrm{CP}$ ). In the following, the case of $15 \mathrm{KHz}$ subcarrier spacing is investigated, which results in 1 slot per subframe [2].

The resource grid width is equal to the number of OFDM/ SEFDM symbols within a subframe, while the height corresponds to the number of resource blocks. For the case of original 5G NR frame with normal CP, the width is 14 symbols [1] and the number of resource blocks depends on the available bandwidth. Each resource block for OFDM has total width equal to one slot in time and total height equal to 12 subcarriers of $15 \mathrm{kHz}$ subcarriers spacing each, which is equal to $180 \mathrm{kHz}$. If SEFDM symbols are used instead of OFDM, then instead of 12 subcarriers per resource block, $\lfloor 12 / \alpha\rfloor$ subcarriers are used and the subcarriers spacing is $(15 \times \alpha) \mathrm{kHz}$ resulting in the same height for OFDM $(180 \mathrm{kHz})$. Fig. 1 demonstrates the resource block design for $\alpha=0.8$, where each resource block contains 15 subcarriers of subcarriers spacing equals to $12 \mathrm{kHz}$.

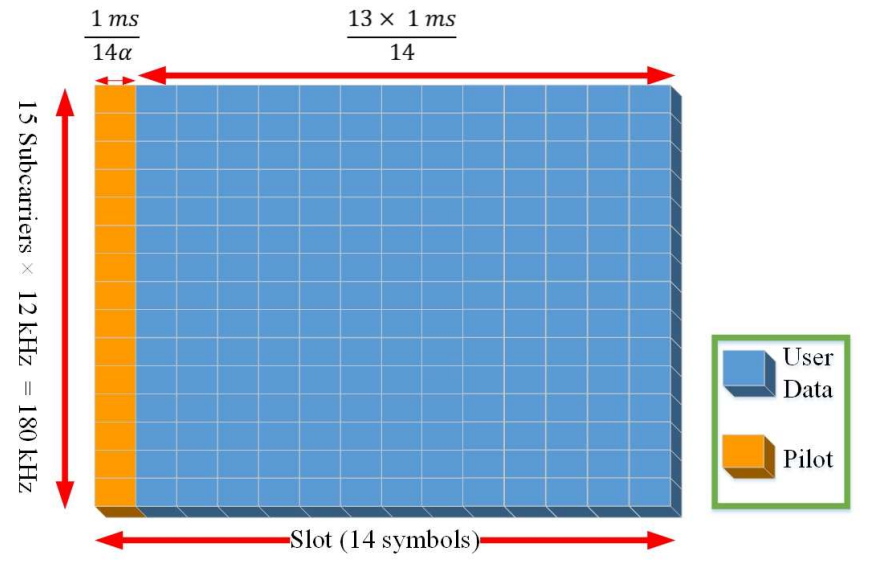

Fig. 1: 5G NR SEFDM-based resource block content for $\alpha=$ 0.8 .

One type of the reference signals sent in each subframe is cell-specific reference (CSR), also known as pilot signals, which is used to estimate the CFR. For the special case of SEFDM signalling format, the pilot is sent over all subcarriers of the first SEFDM symbol as shown in Fig. 1. However, to obtain the channel response without ICI effect, the pilot symbol duration will be longer than the rest SEFDM symbols used to transmit data by a factor of $(1-\alpha) / \alpha$ [15]. Therefore, if each column is of duration $(1 / 14) \mathrm{ms}$, then the first column of each subframe, is of duration $(1 / 14 \alpha) \mathrm{ms}$.

After resource grid mapping, the OFDM/ SEFDM signal generation operates in the resource grid, where it takes one column in a time. The ease and accuracy of OFDM signals generation using an IFFT was one of the main reasons to OFDM being adopted in LTE (4G) cellular network standards [18]. The next section will look at SEFDM signals generation using a single IFFT.

\section{B. IFFT Based SEFDM Transmitter}

In this work, the case of $20 \mathrm{MHz}$ bandwidth is investigated. According to [1], the number of resource blocks for this case is 100 . Thus, the number of subcarriers is 1200 or 1500 for OFDM symbol or SEFDM symbol $(\alpha=0.8)$, respectively. The IFFT size is 2048, where at its input the data is packed and reordered. In NR resource blocks, no explicit DC subcarrier is reserved and the presence or absence of it is known to the receiver. In this work, the DC subcarrier is not present, as no special handling of the DC subcarrier at the receiver has been specified yet by the standards of [2].

If the IFFT is used for OFDM modulation, then all samples at the output of the IFFT will be taken to a parallel to serial $(\mathrm{P} / \mathrm{S})$ converter, then $\mathrm{CP}$ will be added before processing the signal by the analog to digital (ADC) converter. However, for SEFDM signal generation, the output of the IFFT is adjustable to generate the SEFDM data signals, the pilot symbol and continuous $\mathrm{CP}$ as explained below. 


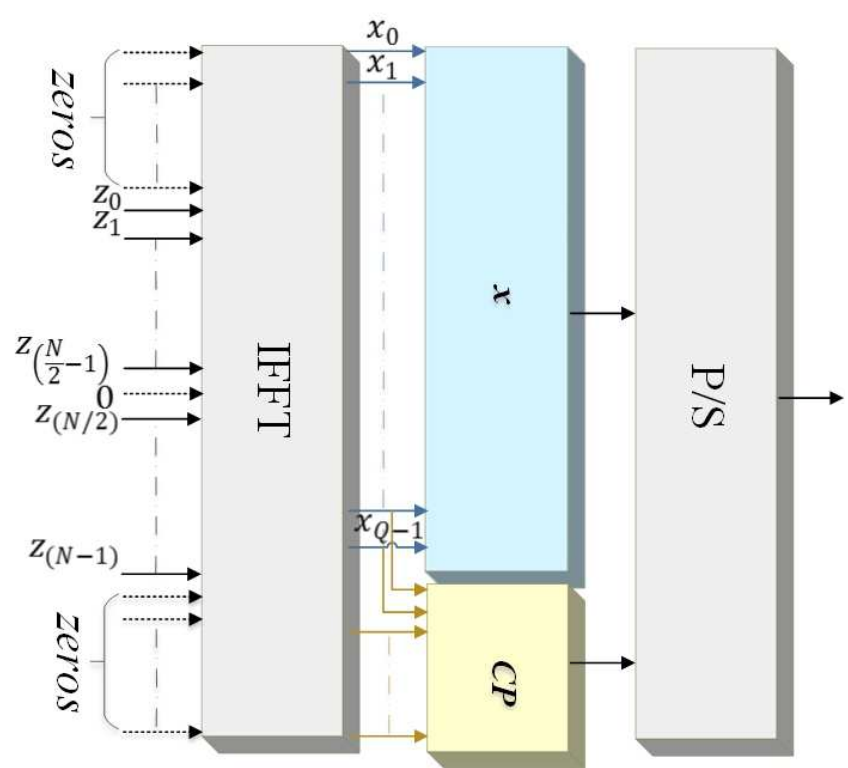

Fig. 2: SEFDM signal generation with continuous $\mathrm{CP}$ via a single IFFT.

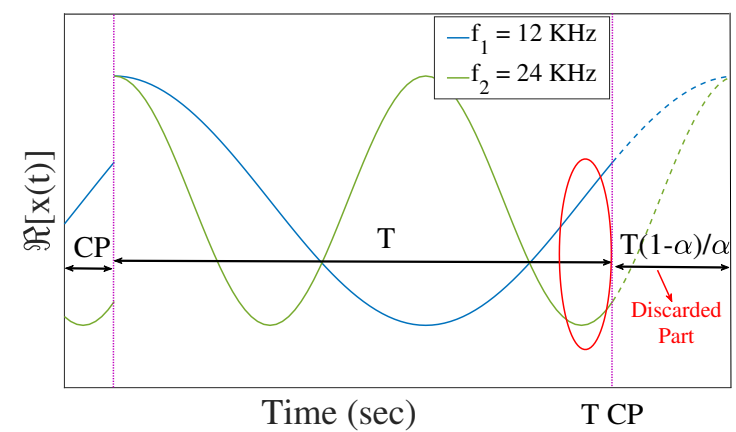

(a) Non-continuous CP.

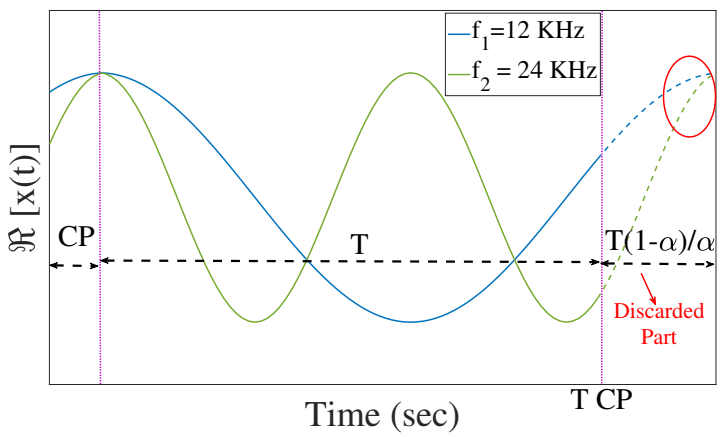

(b) Continuous $\mathrm{CP}$.

Fig. 3: The real part of $\mathrm{x}(\mathrm{t})$ in the time domain for $f_{1}=12$ $\mathrm{kHz}, f_{2}=24 \mathrm{kHz}$ with $\alpha=0.8$.

1) SEFDM data signals: To generate the SEFDM signal $\mathbf{x}$, as shown in Fig. 2, the input to the IFFT is the reordered 1500 complex symbols $\mathbf{z}$, with $\alpha=0.8$. At the output of the IFFT, the first $\lceil 2048 \times \alpha\rceil=1639$ samples are taken while the rest are discarded, where $\lceil$.$\rceil is the ceiling function. The discarded$

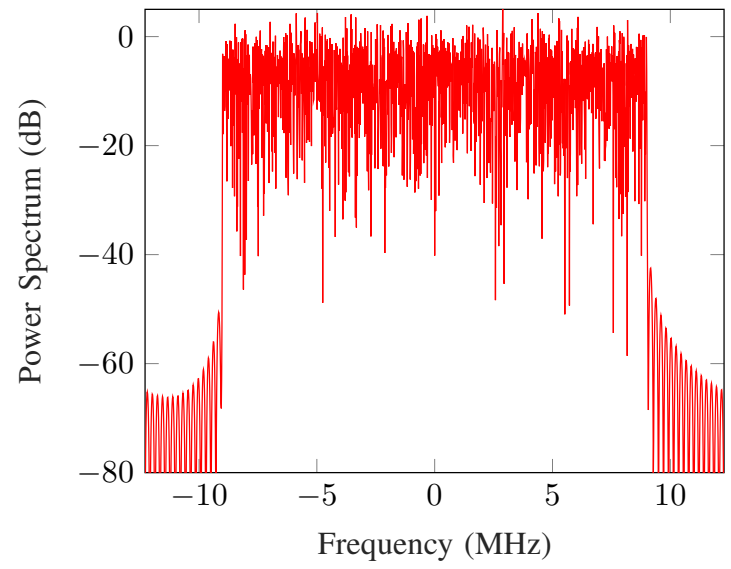

(a) Non-continuous CP.

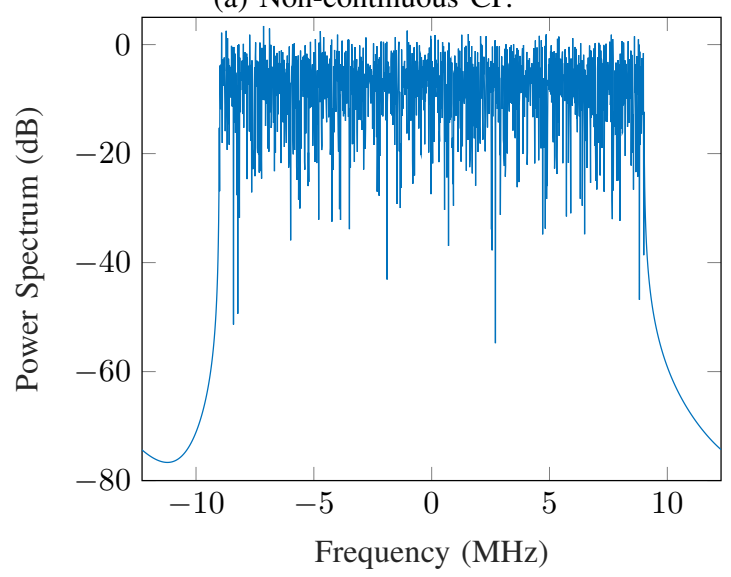

(b) Continuous $\mathrm{CP}$.

Fig. 4: The spectrum of $\mathrm{x}(\mathrm{t})$ for $20 \mathrm{MHz}$ bandwidth with $\alpha=$ 0.8 and different $\mathrm{CP}$ addition cases.

samples at the IFFT output affect the subcarriers compression in SEFDM [17].

2) Continuous cyclic prefix: A guard band or $\mathrm{CP}$ of a duration longer than the channel delay spread of a wireless multipath channel is used to protect the transmitted symbols from inter-symbol interference (ISI) [16]. CP is chosen instead of guard band in $5 \mathrm{G}$ because $\mathrm{CP}$ maintains the transmitted symbol continuity. Hence, the circular convolution in time domain is transferred into multiplication in frequency domain, which allows using a one-tap equaliser to equalise the channel effect. Thus, the property of continuity has to be maintained for SEFDM.

In OFDM, the CP of duration $d$ samples, is created by preceding the symbol with a copy of the last $d$ samples of that same symbol. However, if the same method is followed to create the CP of SEFDM symbols, it will result in a noncontinuous $\mathrm{CP}$ as shown in Fig. 3a, for the first two SEFDM $(\alpha=0.8)$ symbols $\left(f_{1}=12 \mathrm{kHz}, f_{2}=24 \mathrm{kHz}\right)$. To overcome this problem, the CP samples (the samples inside the red circle in the figure) have to be taken from the last $d$ discarded samples of the IFFT as shown in Fig. 2. The effect of this can be seen 

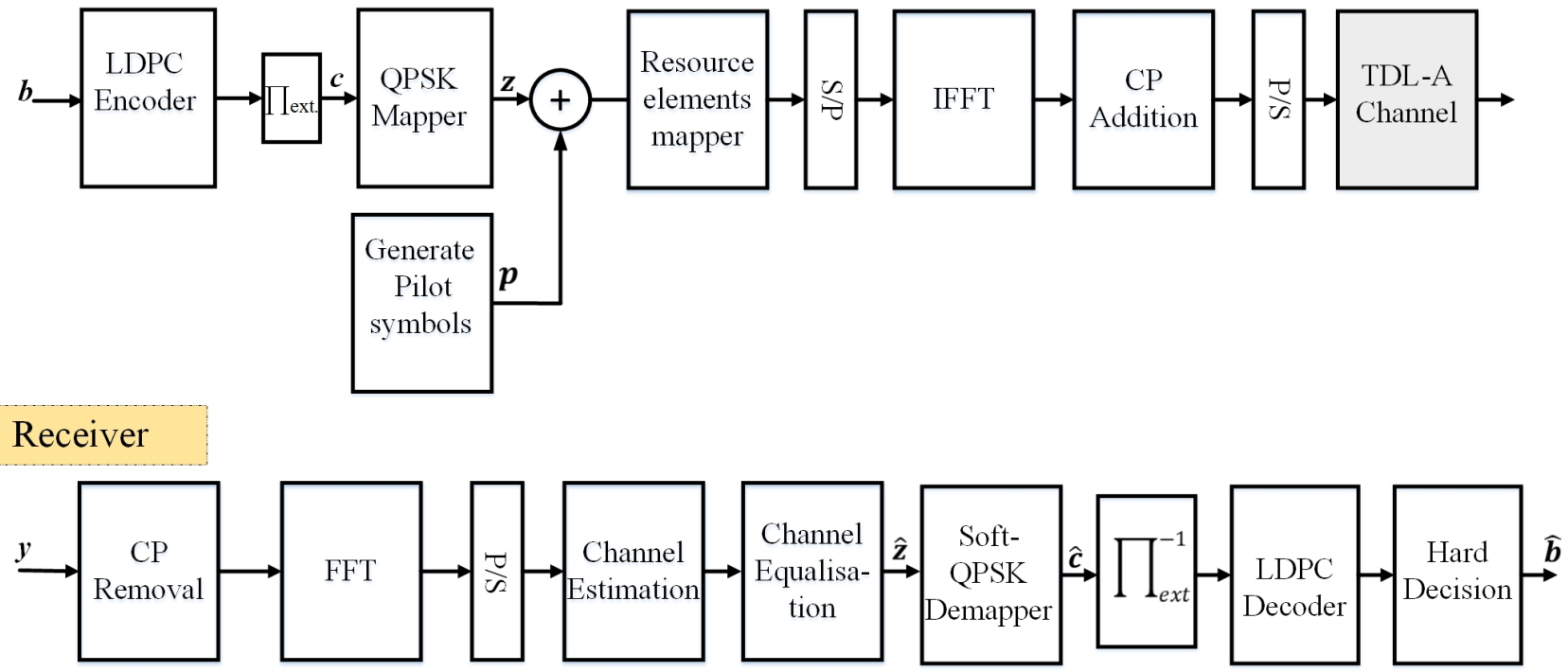

Fig. 5: High level transceiver descriptive design for 5G NR SEFDM-based system.

in Fig. 3b, where the CP is taken from the discarded part (presented by dashed lines).

Furthermore, generating CP properly for SEFDM signals improves the overall system performance as it reduces the out-of-band emission (OOBE) level compared to the noncontinuous case. Fig. 4 demonstrates the normalized spectrum of the SEFDM symbol generated above, after adding the $\mathrm{CP}$ and the P/S stage of the transmitter for both cases.

3) Pilot generation: This is an extension of our recent work of [15], which reports the performance of the newly developed pilot design, without looking at the generation of these pilot symbols using the same IFFT for data generation. The pilot symbols p, from the first column of the resource grid in Fig. 1, are the input to the IFFT in a similar way to the data symbols $\mathbf{z}$. At the output of the IFFT, in contrary to SEFDM data symbols generation, all samples are taken resulting in an OFDM pilot $\mathbf{s}$, with the same number of subcarriers and frequency spacing of the SEFDM signal $\mathbf{x}$. This results in an overhead of $\Gamma((1-$ $\alpha) / \alpha) \times Q\rceil$ samples for the pilot symbol compared to SEFDM data symbols [15]. The CP of the pilot symbol is added directly because the pilot is an OFDM symbol.

\section{Performance Analysis}

To prove the validity of the suggested SEFDM transmission design, a 5G-NR signal transmission scenario is simulated. The scenario is for a specific modulation order (QPSK) and coding rate (1/3), $20 \mathrm{MHz}$ bandwidth and subcarrier spacing $15 \mathrm{KHz}$, $12 \mathrm{KHz}$ for $\alpha=1$ and 0.8 , respectively. As the main focus of this work is at the transmitter, the receiver simulated here is similar to that of OFDM, without sophisticated interference cancellation. However, with higher compression level (i.e. lower $\alpha$ ) and/or higher modulation order, the interference cancellation reported in [8] [19] becomes necessary.

\section{A. Design Methodology}

In this section, we employ a single IFFT to generate $5 \mathrm{G}$ NR SEFDM-based frames, which will be transmitted over a tapped delay line (TDL) channel. Fig. 5 illustrates descriptive high level design of the modelled transceiver. Starting from the far left, first the message bits are encoded by a low-density parity check (LDPC) encoder of a coding rate $R_{c}=1 / 3$. Then, the coded bits are interleaved and mapped to QPSK complex symbols. Simultaneously, QPSK pilot symbols are generated by the gold sequence [20]. Then both pilot and data symbols are mapped into the resource blocks similar to Fig. 1., where the number of resource blocks within the resource grid is 100 for the case of $20 \mathrm{MHz}$ bandwidth. The IFFT in Fig. 2 is used for OFDM/ SEFDM $(\alpha=0.8)$ modulation and a normal CP of length $(7 \%)$ is added to each symbol. The resultant samples are sent over a TDL $5 \mathrm{G}$ channel model of type (D), which has been defined in the new 5G ETSI standards [21] and it represents a line of sight (LOS) channel. The first tap of the TDL-D channel follows a Ricean distribution with a K-factor=7 dB and the rest of the taps follow Rayleigh distribution (given in Table 7.7.2-4 of the standards [21]). The channel simulation parameters are given in Table I.

TABLE I: Channel simulation parameters.

\begin{tabular}{|l|l|}
\hline Parameter & Value \\
\hline Channel delay profile & TDL-D \\
Delay spread & $300 \mathrm{~ns}$ \\
User terminal velocity & $30 \mathrm{~km} /$ hour \\
Maximum Doppler shift & $111.2 \mathrm{~Hz}$ \\
K-factor & $7 \mathrm{~dB}$ \\
\hline
\end{tabular}


At the receiver, firstly, resource elements demapping is perfromed. The pilot is processed to acquire the CFR, where the CP samples distorted by ISI are discarded and pilot samples are demodulated by means of an FFT, which has the exact arrangement of the IFFT at the transmitter. The output symbols from the FFT are used to estimate the CFR [15]. Consequently, the rest of the symbols in a subframe are processed by removing the $\mathrm{CP}$ and demodulation via the FFT, followed by one-tap equaliser using the estimated CFR to equalise the channel effect. Then, the equalised symbols go through a soft QPSK demapper followed by an inverse interleaver and LDPC decoder to obtain the estimated message bits. For this system, the bit error rate (BER) results are simulated and throughput is calculated in the following.

\section{B. BER Results}

Fig. 6 illustrates the BER performance versus energy per bit $\left(E_{b}\right)$ over noise power spectral density $\left(N_{0}\right)$ in $\mathrm{dB}$, for 5G OFDM-based systems and 5G SEFDM-based systems with $(\alpha=0.8)$ continuous $\mathrm{CP}$.

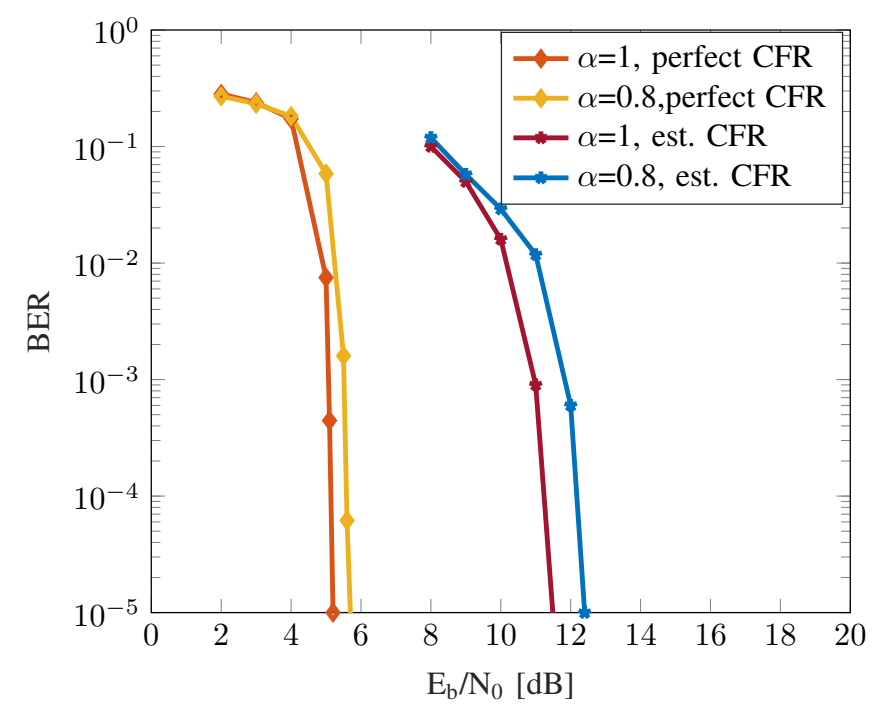

Fig. 6: BER results for 5G SEFDM and OFDM based with perfect and estimated CFR.

Results show that a spectral efficiency can be gained for SEFDM at the expense of an error penalty just below $1 \mathrm{~dB}$. A more sophisticated interference canceller, if used, can further reduce the penalty of SEFDM compared to OFDM at the expense of receiver complexity [5], [8]. Furthermore, the results of both OFDM and SEFDM degrades relative to the results with perfect knowledge of the CFR.

\section{Throughput Calculation}

The throughput $(\eta)$ is calculated by evaluating the total number of subcarriers in a subframe multiplied by the number of bits per modulation symbol, over the subframe duration, such as

$$
\eta=\frac{\log _{2}(M) \times N_{s} \times N}{\frac{(13+1 / \alpha) \times 10^{-3}}{14}},
$$

where $N_{s}$ is the number of symbols per subframe, which is 14 for this case, and $M$ is the constellation order (e.g. $M=4$ for QPSK). For instance, if the channel bandwidth is $20 \mathrm{MHz}$, then number of subcarriers per resource grid is 1200 for $\alpha=1$ and 1500 when $\alpha=0.8$. The throughput using the parameters of the system simulated above for the case of OFDM is 33.60 Mbps and 41.26 Mbps for SEFDM $\alpha=0.8$.

Using SEFDM in the NR $5 \mathrm{G}$ is beneficial in terms of throughput. For example the case above allows an increase in throughput by a factor of $22.8 \%$ compared to OFDM. The effective throughput is calculated by taking into consideration the overhead used for channel estimation, synchronisation and the redundant bits added by channel coding...etc. In both SEFDM and OFDM cases, the overhead percentage is the same.

A higher throughput can be achieved by compressing the subcarriers further, through using a lower value of $\alpha$. However, in this case the error penalty will be higher than the reported results and such case is the subject of further studies.

\section{CONCLUSIONS}

In this work, we presented a new method to generate $5 \mathrm{G} \mathrm{NR}$ SEFDM-based frames via a single adjustable IFFT. This single IFFT is shown to be capable of generating SEFDM symbols, as well as continuous cyclic prefix and an OFDM pilot that has the same subcarriers spacing of the SEFDM symbols. To provide significant insight to the proposed SEFDM signal generation, the single IFFT efficiency is shown by system modelling and simulations for $5 \mathrm{G}$ frames transmission over TDL-A channel. Results show that for the case of $\alpha=0.8$, with a less than $1 \mathrm{~dB}$ error penalty, SEFDM can enhance the throughput by a factor of $22.8 \%$ compared to OFDM. The error penalty can be reduced even more and further subcarriers compression to increase the throughput can be achieved by using sophisticated interference cancellers at the receiver as was shown earlier in related previous work on SEFDM.

\section{ACKNOWLEDGMENT}

We are grateful to UCL for funding Hedaia Ghannam's PhD studies through the Overseas Research Student Award (ORS) and the UCL Dean of Engineering Sciences Award.

\section{REFERENCES}

[1] "3rd Generation Partnership Project; Technical Specification Group Radio Access Network; Study on New Radio (NR) access technology (Release 14)," 3GPP, Tech. Rep., 062017.

[2] "Characteristics template for NR RIT of 5G (Release 15 and beyond)," 3GPP, Tech. Rep., 2017.

[3] M. Rodrigues and I. Darwazeh, "A Spectrally Efficient Frequency Division Multiplexing Based Communications System," in Proc. 8th Int. OFDM Workshop, Hamburg, Nov. 2003, pp. 48-49.

[4] F. Luo and C. Zhang, Signal Processing for 5G: Algorithms and Implementations, ser. Wiley - IEEE. Wiley, 2016.

[5] K. Park, H. Kim, A. Lee, D. Kang, and W. Oh, "Iterative frequencydomain inter-carrier interference cancellation for coded SEFDM," Electronics Lett, vol. 53, no. 19, pp. 1333-1335, 2017.

[6] S. Peng, A. Liu, K. Wang, and X. Liang, "PAPR Reduction of Multicarrier Faster-Than-Nyquist Signals with Partial Transmit Sequence," IEEE Access, vol. 5, pp. 24 931-24 937, 2017. 
[7] S. Isam and I. Darwazeh, "Peak to Average Power Ratio Reduction in Spectrally Efficient FDM Systems," in 2011 18th ICT, May 2011, pp. 363-368.

[8] H. Ghannam and I. Darwazeh, "SEFDM over satellite systems with advanced interference cancellation," IET Communications, vol. 12, no. 1, pp. 59-66, 2018.

[9] T. Xu and I. Darwazeh, "Transmission Experiment of Bandwidth Compressed Carrier Aggregation in a Realistic Fading Channel," IEEE Trans. Veh. Technol., 2016.

[10] T. Xu, S. Mikroulis, J. E. Mitchell, and I. Darwazeh, "Bandwidth Compressed Waveform for 60-GHz Millimeter-Wave Radio over Fiber Experiment," J. of Lightwave Technol., vol. 34, no. 14, pp. 3458-3465, July 2016.

[11] Y. Wang, Y. Zhou, T. Gui, K. Zhong, X. Zhou, L. Wang, A. P. T. Lau, C. Lu, and N. Chi, "SEFDM Based Spectrum Compressed VLC System Using RLS Time-domain Channel estimation and ID-FSD Hybrid Decoder," in ECOC 2016; 42nd, Sept 2016, pp. 1-3.

[12] D. Nopchinda, T. Xu, R. Maher, B. C. Thomsen, and I. Darwazeh, "Dual Polarization Coherent Optical Spectrally Efficient Frequency Division Multiplexing," IEEE Photon. Technol. Lett, vol. 28, no. 1, pp. 83-86, Jan 2016.

[13] J. Zhou, Y. Qiao, Z. Yang, M. Guo, and X. Tang, "Capacity Limit for Faster-than-Nyquist Non-Orthogonal Frequency-Division Multiplexing Signaling," in Scientific Reports, 2017.

[14] T. Xu and I. Darwazeh, "Non-orthogonal narrowband internet of things: A design for saving bandwidth and doubling the number of connected devices," IEEE Internet of Things Journal, vol. 5, no. 3, pp. 2120-2129, June 2018.

[15] H. Ghannam and I. Darwazeh, "Robust channel estimation methods for spectrally efficient FDM systems," in VTC 2018 workshop; Technology Trials and Proof-of-Concept Activities for 5 G and Beyond 2018 (TPoC5G 2018) (accepted), June 2018.

[16] B. Muquet, Z. Wang, G. B. Giannakis, M. de Courville, and P. Duhamel, "Cyclic prefixing or zero padding for wireless multicarrier transmissions?" IEEE Trans on Commun, vol. 50, no. 12, pp. 2136-2148, Dec 2002.

[17] P. N. Whatmough, M. R. Perrett, S. Isam, and I. Darwazeh, "VLSI Architecture for a Reconfigurable Spectrally Efficient FDM Baseband Transmitter," IEEE Trans. Circuits Syst. I, Reg. Papers, vol. 59, no. 5, pp. 1107-1118, May 2012.

[18] S. Weinstein and P. Ebert, "Data transmission by frequency-division multiplexing using the discrete Fourier transform," IEEE Trans on Commun Tech, vol. 19, no. 5, pp. 628-634, October 1971.

[19] T. Xu and I. Darwazeh, "A joint waveform and precoding design for nonorthogonal multicarrier signals," in 2017 IEEE Wireless Communications and Networking Conference (WCNC), March 2017, pp. 1-6.

[20] J. Ahn, K. Kim, D. Lee, and J. Lee, "Reference signal generation using gold sequences," Sep. 23 2009, eP Patent App. EP20,090,155,364.

[21] "5G; Study on channel model for frequencies from 0.5 to $100 \mathrm{GHz}$ (3GPP TR 38.901 version 14.0.0 Release 14)," ETSI, Tech. Rep., 05 2017. 